\title{
Polyphase matrix characterization of framelets on local fields of positive characteristic
}

\author{
F. A. Shah \\ Department of Mathematics, \\ University of Kashmir, India \\ email: fashah79@gmail.com
}

\author{
M. Y. Bhat \\ Department of Mathematics, \\ Central University of Jammu, India \\ email: gyounusg@gmail.com
}

\begin{abstract}
An important tool for the construction of framelets on local fields of positive characteristic using unitary extension principle was presented by Shah and Debnath [Tight wavelet frames on local fields, Analysis, 33 (2013), 293-307]. In this article, we continue the study of framelets on local fields and present a polyphase matrix characterization of framelets generated by the extension principle.
\end{abstract}

\section{Introduction}

Along with the study of wavelet bases, there had been a continuing research effort in the study of tight wavelet frames (framelets) and have gained considerable popularity in recent times, primarily due to their substantiated applications in diverse and widespread fields of science and engineering. A framelet is a generalization of an orthonormal wavelet basis by introducing redundancy into a wavelet system. By sacrificing orthonormality and allowing redundancy, the framelets become much easier to construct than the orthonormal wavelets. The main tool for construction and characterization of wavelet frames are

2010 Mathematics Subject Classification: 42C40, 42C15, 43A70, 11S85

Key words and phrases: wavelet frame, framelet, wavelet mask, polyphase matrix, local field, extension principle, Fourier transform 
the several extension principles, the unitary extension principle and oblique extension principle as well as their generalized versions, the mixed unitary extension principle and the mixed oblique extension principle. They provide sufficient conditions for constructing tight and dual wavelet frames for any given refinable function which generates a multiresolution analysis (MRA). These essential methods were firstly introduced by Ron and Shen in [8] and in the fundamental work of Daubechies et al. [2] for scalar refinable functions. The resulting tight wavelet frames are based on a multiresolution analysis, and the generators are often called framelets. To mention only a few references on tight wavelet frames, the reader is referred to [3], [5]-[7] and many references therein.

A field $\mathrm{K}$ equipped with a topology is called a local field if both the additive $\mathrm{K}^{+}$and multiplicative groups $\mathrm{K}^{*}$ of $\mathrm{K}$ are locally compact Abelian groups. For example, any field endowed with the discrete topology is a local field. For this reason we consider only non-discrete fields. The local fields are essentially of two types (excluding the connected local fields $\mathbb{R}$ and $\mathbb{C}$ ). The local fields of characteristic zero include the p-adic field $\mathbb{Q}_{p}$. Examples of local fields of positive characteristic are the Cantor dyadic group and the Vilenkin p-groups. Even though the structures and metrics of local fields of zero and positive characteristics are similar, their wavelet and multiresolution analysis theory are quite different.

The local field $\mathrm{K}$ is a natural model for the structure of wavelet frame systems, as well as a domain upon which one can construct wavelet basis functions. There is a substantial body of work that has been concerned with the construction of wavelets on local fields or more generally on local fields of positive characteristic. For example, L. Benedetto and J. Benedetto [1] developed a wavelet theory for local fields and related groups. They did not develop the multiresolution analysis (MRA) approach, their method is based on the theory of wavelet sets and only allows the construction of wavelet functions whose Fourier transforms are characteristic functions of some sets. Jiang et al. [4] pointed out a method for constructing orthogonal wavelets on local field $\mathrm{K}$ with a constant generating sequence and derived necessary and sufficient conditions for a solution of the refinement equation to generate a multiresolution analysis of $\mathrm{L}^{2}(\mathrm{~K})$. Subsequently, tight wavelet frames on local fields of positive characteristic were constructed by Shah and Debnath [15] using extension principles. More precisely, they provide a sufficient condition for finite number of functions $\left\{\psi_{1}, \psi_{2}, \ldots, \psi_{\mathrm{L}}\right\}$ to form a tight wavelet frame for $\mathrm{L}^{2}(\mathrm{~K})$ and established a complete characterization of tight wavelet frames on 
local fields by virtue of the modulation matrix $\mathcal{M}(\xi)=\left\{h_{\ell}(\mathfrak{p} \xi+\mathfrak{p u}(k))\right\}_{\ell, k=0}^{q-1}$ formed by the framelet symbols $m_{\ell}(\xi), \ell=0,1, \ldots, L$ associated with the scaling function $\phi(x)$ and basic wavelets $\psi_{\ell}(x), 1 \leq \ell \leq$ L. The characterizations of tight wavelet frames on local fields were completely established by Shah and Abdullahb [12] by virtue of two basic equations in the Fourier domain. These studies were continued by Shah and his colleagues in [9]-[14], where they have provided some algorithms for constructing wave packet frames, framelet packets, semi-orthogonal wavelet frames and periodic wavelet frames on local fields of positive characteristic.

Drawing inspiration from the construction of framelets on local fields of positive characteristic, in this article, we firstly provide the polyphase representation of the framelet symbols $m_{\ell}(\xi), \ell=0,1, \ldots, L$ and then, establish a complete characterization of framelets on local fields in terms of the polyphase matrix $\mathcal{P}(\xi)=\left\{f_{r}^{\ell}(\xi)\right\}_{\ell, k=0}^{q-1}$ formed by the polyphase components $f_{r}^{\ell}, r=0,1, \ldots, q-1$ of the framelet symbols $m_{\ell}(\xi)$.

The rest of this paper is organized as follows. In Section 2, we discuss some preliminary facts about local fields of positive characteristic and review some major concepts concerning framelets on local fields. In Section 3, we prove the main result of our paper, shows that a unitary polyphase matrix leads to a tight wavelet frame on local fields of positive characteristic.

\section{Preliminaries on local fields}

Let $\mathrm{K}$ be a field and a topological space. Then $\mathrm{K}$ is called a local field if both $\mathrm{K}^{+}$and $\mathrm{K}^{*}$ are locally compact Abelian groups, where $\mathrm{K}^{+}$and $\mathrm{K}^{*}$ denote the additive and multiplicative groups of $\mathrm{K}$, respectively. If $\mathrm{K}$ is any field and is endowed with the discrete topology, then $K$ is a local field. Further, if $K$ is connected, then $\mathrm{K}$ is either $\mathbb{R}$ or $\mathbb{C}$. If $\mathrm{K}$ is not connected, then it is totally disconnected. Hence by a local field, we mean a field $\mathrm{K}$ which is locally compact, non-discrete and totally disconnected. The p-adic fields are examples of local fields. We use the notation of the book by Taibleson [16]. In the rest of this paper, we use the symbols $\mathbb{N}, \mathbb{N}_{0}$ and $\mathbb{Z}$ to denote the sets of natural, nonnegative integers and integers, respectively.

Let $\mathrm{K}$ be a local field. Let $\mathrm{d} x$ be the Haar measure on the locally compact Abelian group $\mathrm{K}^{+}$. If $\alpha \in \mathrm{K}$ and $\alpha \neq 0$, then $\mathrm{d}(\alpha x)$ is also a Haar measure. Let $\mathrm{d}(\alpha x)=|\alpha| \mathrm{d} x$. We call $|\alpha|$ the absolute value of $\alpha$. Moreover, the map $x \rightarrow|x|$ has the following properties:

(a) $|x|=0$ if and only if $x=0$; 
(b) $|x y|=|x||y|$ for all $x, y \in K$;

(c) $|x+y| \leq \max \{|x|,|y|\}$ for all $x, y \in K$.

Property (c) is called the ultrametric inequality. The set $\mathfrak{D}=\{x \in \mathrm{K}:|x| \leq 1\}$ is called the ring of integers in K. Define $\mathfrak{B}=\{x \in \mathrm{K}:|x|<1\}$. The set $\mathfrak{B}$ is called the prime ideal in $\mathrm{K}$. The prime ideal in $\mathrm{K}$ is the unique maximal ideal in $\mathfrak{D}$ and hence as result $\mathfrak{B}$ is both principal and prime. Since the local field $\mathrm{K}$ is totally disconnected, so there exist an element of $\mathfrak{B}$ of maximal absolute value. Let $\mathfrak{p}$ be a fixed element of maximum absolute value in $\mathfrak{B}$. Such an element is called a prime element of $\mathrm{K}$. Therefore, for such an ideal $\mathfrak{B}$ in $\mathfrak{D}$, we have $\mathfrak{B}=\langle\mathfrak{p}\rangle=\mathfrak{p} \mathfrak{D}$. As it was proved in [16], the set $\mathfrak{D}$ is compact and open. Hence, $\mathfrak{B}$ is compact and open. Therefore, the residue space $\mathfrak{D} / \mathfrak{B}$ is isomorphic to a finite field $\mathrm{GF}(\mathrm{q})$, where $\mathrm{q}=\mathrm{p}^{\mathrm{k}}$ for some prime $\mathrm{p}$ and $\mathrm{k} \in \mathbb{N}$.

Let $\mathfrak{D}^{*}=\mathfrak{D} \backslash \mathfrak{B}=\{x \in \mathrm{K}:|x|=1\}$. Then, it can be proved that $\mathfrak{D}^{*}$ is a group of units in $\mathrm{K}^{*}$ and if $x \neq 0$, then we may write $x=\mathfrak{p}^{k} \chi^{\prime}, \chi^{\prime} \in \mathfrak{D}^{*}$. For a proof of this fact we refer to [16]. Moreover, each $\mathfrak{B}^{k}=\mathfrak{p}^{k} \mathfrak{D}=\left\{x \in \mathrm{K}:|x|<\mathrm{q}^{-\mathrm{k}}\right\}$ is a compact subgroup of $\mathrm{K}^{+}$and usually known as the fractional ideals of $\mathrm{K}^{+}$. Let $\mathcal{U}=\left\{\mathrm{a}_{i}\right\}_{\mathfrak{i}=0}^{\mathrm{q}-1}$ be any fixed full set of coset representatives of $\mathfrak{B}$ in $\mathfrak{D}$, then every element $\chi \in K$ can be expressed uniquely as $\chi=\sum_{\ell=K}^{\infty} c_{\ell} p^{\ell}$ with $\mathfrak{c}_{\ell} \in \mathcal{U}$. Let $\chi$ be a fixed character on $\mathrm{K}^{+}$that is trivial on $\mathfrak{D}$ but is nontrivial on $\mathfrak{B}^{-1}$. Therefore, $\chi$ is constant on cosets of $\mathfrak{D}$ so if $y \in \mathfrak{B}^{k}$, then $\chi_{y}(x)=\chi(y x), x \in K$. Suppose that $\chi_{\mathfrak{u}}$ is any character on $\mathrm{K}^{+}$, then clearly the restriction $\chi_{\mathfrak{u}} \mid \mathfrak{D}$ is also a character on $\mathfrak{D}$. Therefore, if $\left\{\mathfrak{u}(n): n \in \mathbb{N}_{0}\right\}$ is a complete list of distinct coset representative of $\mathfrak{D}$ in $\mathrm{K}^{+}$, then, as it was proved in [16], the set $\left\{\chi_{\mathfrak{u}(\mathfrak{n})}: \mathfrak{n} \in \mathbb{N}_{0}\right\}$ of distinct characters on $\mathfrak{D}$ is a complete orthonormal system on $\mathfrak{D}$.

The Fourier transform $\hat{f}$ of a function $f \in L^{1}(K) \cap L^{2}(K)$ is defined by

$$
\hat{f}(\xi)=\int_{K} f(x) \overline{\chi_{\xi}(x)} d x .
$$

It is noted that

$$
\hat{f}(\xi)=\int_{K} f(x) \overline{\chi \xi(\chi)} d x=\int_{K} f(x) \chi(-\xi x) d x .
$$

The properties of the Fourier transform on the local field $K$ are quite similar to those of the Fourier analysis on the real line (See Taibleson [16]). In particular, if $f \in \mathrm{L}^{1}(\mathrm{~K}) \cap \mathrm{L}^{2}(\mathrm{~K})$, then $\hat{\mathrm{f}} \in \mathrm{L}^{2}(\mathrm{~K})$ and $\|\hat{\mathrm{f}}\|_{2}=\|\mathrm{f}\|_{2}$.

We now impose a natural order on the sequence $\{\mathfrak{u}(\mathfrak{n})\}_{\mathfrak{n}=0}^{\infty}$. We have $\mathfrak{D} / \mathfrak{B} \cong$ $\mathrm{GF}(\mathrm{q})$ where $\mathrm{GF}(\mathrm{q})$ is a c-dimensional vector space over the field $\mathrm{GF}(\mathrm{p})$. We 
choose a set $\left\{1=\zeta_{0}, \zeta_{1}, \zeta_{2}, \ldots, \zeta_{c-1}\right\} \subset \mathfrak{D}^{*}$ such that $\operatorname{span}\left\{\zeta_{\mathbf{j}}\right\}_{j=0}^{c-1} \cong \mathrm{GF}(\mathbf{q})$. For $\mathrm{n} \in \mathbb{N}_{0}$ satisfying

$0 \leq n<q, \quad n=a_{0}+a_{1} p+\cdots+a_{c-1} p^{c-1}, 0 \leq a_{k}<p$, and $k=0,1, \ldots, c-1$, we define

$$
u(n)=\left(a_{0}+a_{1} \zeta_{1}+\cdots+a_{c-1} \zeta_{c-1}\right) \mathfrak{p}^{-1} .
$$

Also, for $n=b_{0}+b_{1} q+b_{2} q^{2}+\cdots+b_{s} q^{s}, n \in \mathbb{N}_{0}, 0 \leq b_{k}<q, k=$ $0,1,2, \ldots, s$, we set

$$
u(n)=u\left(b_{0}\right)+u\left(b_{1}\right) \mathfrak{p}^{-1}+\cdots+u\left(b_{s}\right) \mathfrak{p}^{-s} .
$$

This defines $\mathfrak{u}(n)$ for all $n \in \mathbb{N}_{0}$. In general, it is not true that $\mathfrak{u}(m+n)=$ $u(m)+u(n)$. But, if $r, k \in \mathbb{N}_{0}$ and $0 \leq s<q^{k}$, then $u\left(r q^{k}+s\right)=u(r) p^{-k}+u(s)$. Further, it is also easy to verify that $u(n)=0$ if and only if $n=0$ and $\left\{\mathfrak{u}(\ell)+\mathfrak{u}(k): k \in \mathbb{N}_{0}\right\}=\left\{\mathfrak{u}(k): k \in \mathbb{N}_{0}\right\}$ for a fixed $\ell \in \mathbb{N}_{0}$. Hereafter we use the notation $\chi_{\mathfrak{n}}=\chi_{\mathfrak{u}(\mathrm{n})}, \mathrm{n} \geq 0$.

Let the local field $K$ be of characteristic $p>0$ and $\zeta_{0}, \zeta_{1}, \zeta_{2}, \ldots, \zeta_{c-1}$ be as above. We define a character $\chi$ on $\mathrm{K}$ as follows:

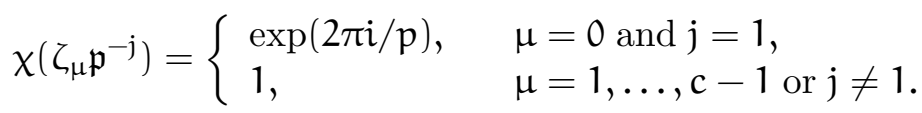

Since $\bigcup_{\mathfrak{j} \in \mathbb{Z}} \mathfrak{p}^{-j} \mathfrak{D}=K$, we can regard $\mathfrak{p}^{-1}$ as the dilation and since $\{\mathfrak{u}(\mathfrak{n}): \mathfrak{n}$ $\left.\in \mathbb{N}_{0}\right\}$ is a complete list of distinct coset representatives of $\mathfrak{D}$ in $K$, the set $\Lambda=\left\{\mathfrak{u}(\mathrm{n}): \mathrm{n} \in \mathbb{N}_{0}\right\}$ can be treated as the translation set. Note that $\Lambda$ is a subgroup of $\mathrm{K}^{+}$and unlike the standard wavelet theory on the real line, the translation set is not a group.

For given $\Psi:=\left\{\psi_{1}, \ldots, \psi_{\mathrm{L}}\right\} \subset \mathrm{L}^{2}(\mathrm{~K})$, define the wavelet system

$$
\mathcal{W}(\psi, j, k):=\left\{\psi_{j, k}^{\ell}: 1 \leq \ell \leq \mathrm{L}, j \in \mathbb{Z}, \mathrm{k} \in \mathbb{N}_{0}\right\}
$$

where $\psi_{j, k}^{\ell}=q^{j / 2} \psi^{\ell}\left(\mathfrak{p}^{-j} \cdot-\mathfrak{u}(k)\right)$. The wavelet system $\mathcal{W}(\psi, j, k)$ is called a framelet system, if there exist positive numbers $0<A \leq B<\infty$ such that

$$
A\|f\|_{2}^{2} \leq \sum_{\ell=1}^{L} \sum_{j \in \mathbb{Z}} \sum_{k \in \mathbb{Z}}\left|\left\langle f, \psi_{j, k}^{\ell}\right\rangle\right|^{2} \leq B\|f\|_{2}^{2}, \quad \text { for all } f \in L^{2}(K) .
$$

The largest $A$ and the smallest B for which (6) holds are called wavelet frame bounds. A wavelet frame is a tight wavelet frame if $A$ and $B$ are chosen such that 
$A=B$ and then generators $\psi_{1}, \psi_{2}, \ldots, \psi_{\mathrm{L}}$ are often referred as tight framelets. If only the right-hand inequality in (6) holds, then the system $\mathcal{W}(\psi, j, k)$ is called a Bessel sequence.

The construction of framelet systems often starts with the construction of a multiresolution analysis (MRA), which is built on refinable functions. A function $\phi \in \mathrm{L}^{2}(\mathrm{~K})$ is called refinable if it satisfies a refinement equation:

$$
\phi(x)=\sqrt{q} \sum_{k \in \mathbb{N}_{0}} h_{k} \phi\left(\mathfrak{p}^{-1} x-u(k)\right),
$$

for some $\left\{h_{k}: k \in \mathbb{N}_{0}\right\} \in l^{2}\left(\mathbb{N}_{0}\right)$. In the frequency domain, (7) can be written as

$$
\hat{\phi}(\xi)=m_{0}(\mathfrak{p} \xi) \hat{\phi}(\mathfrak{p} \xi),
$$

where

$$
m_{0}(\xi)=\frac{1}{\sqrt{q}} \sum_{k \in \mathbb{N}_{0}} h_{k} \overline{\chi_{k}(\xi)},
$$

is an integral periodic function in $\mathrm{L}^{2}(\mathfrak{D})$ and is often called the refinement symbol of $\phi$.

For a refinable function $\phi \in \mathrm{L}^{2}(\mathrm{~K})$ with $\hat{\phi}(0) \neq 0$, let $\mathrm{V}_{0}$ be the closed shift invariant space generated by $\left\{\phi\left(\cdot-u(k): k \in \mathbb{N}_{0}\right\}\right.$ and $V_{j}=\left\{\phi\left(\mathfrak{p}^{-j}-u(k)\right)\right.$ : $\left.k \in \mathbb{N}_{0}\right\}, j \in \mathbb{Z}$. Then, it is proved in [4] that the closed subspaces $\left\{V_{j}: j \in \mathbb{Z}\right\}$ constitutes an MRA for $\mathrm{L}^{2}(\mathrm{~K})$. Recall that an MRA is a family of closed subspaces $\left\{V_{j}: j \in \mathbb{Z}\right\}$ of $L^{2}(K)$ that satisfies (i) $V_{j} \subset V_{j+1}, j \in \mathbb{Z}$; (ii) $\bigcup_{j \in \mathbb{Z}} V_{j}$ is dense in $\mathrm{L}^{2}(\mathrm{~K})$ and (iii) $\bigcap_{\mathrm{j} \in \mathbb{Z}} \mathrm{V}_{j}=\{0\}$. We further assume that

$$
|\hat{\phi}(0)|^{2}=1 \quad \text { and } \quad \lim _{j \rightarrow \infty}\left|\hat{\phi}\left(\mathfrak{p}^{-j} \xi\right)\right|=0 \quad \text { for a.e. } \xi \in K \text {. }
$$

Given an MRA generated by the refinable function $\phi(x)$, one can construct a set of basic tight framelets $\Psi:=\left\{\psi_{1}, \ldots, \psi_{\mathrm{L}}\right\} \subset \mathrm{V}_{1}$ satisfying

$$
\hat{\psi}^{\ell}(\xi)=m_{\ell}(\mathfrak{p} \xi) \hat{\phi}(\mathfrak{p} \xi),
$$

where

$$
m_{\ell}(\xi)=\frac{1}{\sqrt{\mathrm{q}}} \sum_{\mathrm{k} \in \mathbb{N}_{0}} h_{\mathrm{k}}^{\ell} \overline{\chi_{\mathrm{k}}(\xi)}, \quad \ell=1, \ldots, \mathrm{L}
$$

are the integral periodic functions in $\mathrm{L}^{2}(\mathfrak{D})$ and are called the framelet symbols or wavelet masks (see [5]). 
With $m_{\ell}(\xi), \ell=0,1, \ldots L, L \geq q-1$, as the wavelet masks, we formulate the matrix $\mathcal{M}(\xi)$ as:

$$
\mathcal{M}(\xi)=\left(\begin{array}{cccc}
\mathrm{m}_{0}(\xi+\mathfrak{p u}(0)) & \mathrm{m}_{0}(\xi+\mathfrak{p u}(1)) & \ldots & \mathrm{m}_{0}(\xi+\mathfrak{p u}(\mathfrak{q}-1)) \\
\mathrm{m}_{1}(\xi+\mathfrak{p u}(0)) & \mathrm{m}_{1}(\xi+\mathfrak{p u}(1)) & \ldots & \mathrm{m}_{1}(\xi+\mathfrak{p u}(\mathfrak{q}-1)) \\
\vdots & \vdots & \ddots & \vdots \\
\mathrm{m}_{\mathrm{L}}(\xi+\mathfrak{p u}(0)) & \mathrm{m}_{\mathrm{L}}(\xi+\mathfrak{p u}(1)) & \ldots & \mathrm{m}_{\mathrm{L}}(\xi+\mathfrak{p u}(\mathfrak{q}-1))
\end{array}\right) .
$$

The matrix $\mathcal{M}(\xi)$ is called the modulation matrix. Shah and Debnath [15] gave a complete characterization of tight wavelet frames on local fields via extension principles and established a sufficient condition on $\Psi=\left\{\psi_{1}, \ldots, \psi_{\mathrm{L}}\right\}$ such that the resulting wavelet system $\mathcal{W}(\psi, j, k)$ given by (5) forms a tight frame for $L^{2}(K)$. More precisely, the framlet system $\mathcal{W}(\psi, j, k)$ forms a tight framelet frame for $\mathrm{L}^{2}(\mathrm{~K})$ if

$$
\mathcal{M}(\xi) \mathcal{M}^{*}(\xi)=\mathrm{I}_{\mathrm{q}}, \quad \text { for } \quad \text { a.e. } \xi \in \sigma\left(\mathrm{V}_{0}\right)
$$

where $\sigma\left(V_{0}\right):=\left\{\xi \in \mathfrak{D}: \sum_{k \in \mathbb{Z}}|\hat{\phi}(\xi+u(k))|^{2} \neq 0\right\}$.

\section{Polyphase matrix characterization of framelets on local fields}

In this section, we shall first drive the polyphase representation of framelet symbols $m_{\ell}(\xi), \ell=0,1, \ldots, L$ and then establish a complete characterization of tight framelets by means of their polyphase components.

The polyphase representation of the refinement mask $m_{0}(\xi)$ can be derived as

$$
\begin{aligned}
m_{0}(\xi) & =\frac{1}{\sqrt{q}} \sum_{k \in \mathbb{N}_{0}} h_{k} \overline{\chi_{k}(\xi)} \\
& =\frac{1}{\sqrt{q}} \sum_{r=0}^{q-1} \sum_{k \in \mathbb{N}_{0}} h_{\mathfrak{u}(r)+q k} \overline{\chi_{u(r)+q k}(\xi)} \\
& =\frac{1}{\sqrt{q}} \sum_{r=0}^{q-1} \overline{\chi_{u(r)}(\xi)} \sum_{k \in \mathbb{N}_{0}} h_{u(r)+q k} \overline{\chi_{q k}(\xi)} \\
& =\frac{1}{\sqrt{q}} \sum_{r=0}^{q-1} \overline{\chi_{u(r)}(\xi)} f_{r}^{0}(\overline{\chi(q \xi)}),
\end{aligned}
$$


where

$$
f_{r}^{0}(\xi)=\sum_{k \in \mathbb{N}_{0}} h_{u(r)+q k} \overline{x(k)}, \quad r=0,1, \ldots, q-1, x \in K .
$$

Similarly, the framelet symbols $m_{\ell}(\xi), \ell=1,2, \ldots, L$ as defined in equation (12) can be splitted into polyphase components as

$$
m_{\ell}(\xi)=\frac{1}{\sqrt{q}} \sum_{r=0}^{q-1} \overline{\chi_{u(r)}(\xi)} f_{r}^{\ell}(\overline{\chi(q \xi)}),
$$

where

$$
f_{r}^{\ell}(\xi)=\sum_{k \in \mathbb{N}_{0}} h_{u(r)+q k} \overline{x(k)}, \quad r=0,1, \ldots, q-1, x \in K .
$$

With the polyphase components given by equations (15) and (16), we formulate the polyphase matrix $\mathcal{P}(\xi)$ as:

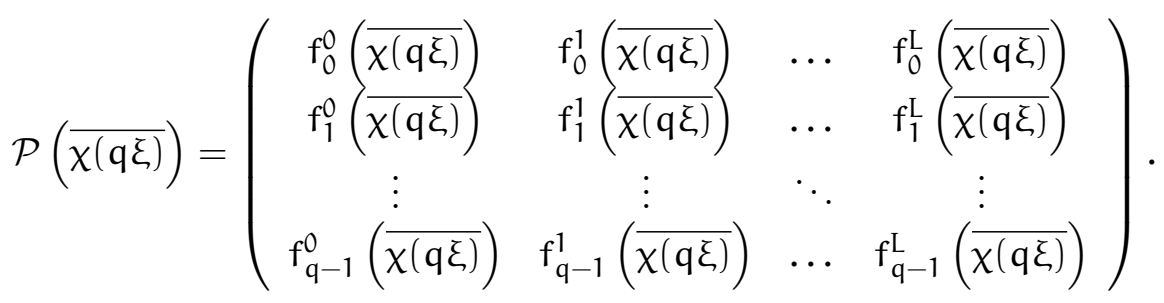

Then, it is clear that

$$
\mathcal{M}(\xi)=\mathcal{S}(\overline{\chi(\xi)}) \mathcal{P}(\overline{\chi(q \xi)})
$$

where

$$
\mathcal{S}(\overline{\chi(\xi)})=\left(\begin{array}{cccc}
\bar{\chi}_{\mathfrak{u}(0)}(\xi+\mathfrak{u}(0)) & \bar{\chi}_{\mathfrak{u}(1)}(\xi+\mathfrak{u}(0)) & \ldots & \bar{\chi}_{\mathfrak{u}(\mathbf{q}-1)}(\xi+\mathfrak{u}(0)) \\
\bar{\chi}_{\mathfrak{u}(0)}(\xi+\mathfrak{u}(1)) & \bar{\chi}_{\mathfrak{u}(1)}(\xi+\mathfrak{u}(1)) & \ldots & \bar{\chi}_{\mathfrak{u}(\mathbf{q}-1)}(\xi+\mathfrak{u}(1)) \\
\vdots & \vdots & \ddots & \vdots \\
\bar{\chi}_{\mathfrak{u}(0)}(\xi+\mathfrak{u}(\mathbf{q}-1)) & \bar{\chi}_{\mathfrak{u}(1)}(\xi+\mathfrak{u}(\mathbf{q}-1)) & \ldots & \bar{\chi}_{\mathfrak{u}(\mathbf{q}-1)}(\xi+\mathfrak{u}(\mathbf{q}-1))
\end{array}\right) .
$$

Since $\mathcal{S}(\overline{\chi(\xi)})$ is unitary matrix, therefore condition (14) is equivalent to

$$
\mathcal{P}(\overline{\chi(q \xi)}) \mathcal{P}(\overline{\chi(q \xi)})^{*}=I_{q} .
$$

For convenience, let $\overline{\chi(\mathrm{q} \xi)}=\zeta$, then the matrix (17) can be rewritten as

$$
\mathcal{P}(\zeta)=\left(\begin{array}{cccc}
f_{0}^{0}(\zeta) & f_{0}^{1}(\zeta) & \cdots & \left.f_{0}^{\mathrm{L}}(\zeta)\right) \\
f_{1}^{0}(\zeta) & f_{1}^{1}(\zeta) & \cdots & \left.f_{1}^{\mathrm{L}}(\zeta)\right) \\
\vdots & \vdots & \ddots & \vdots \\
f_{\mathrm{q}-1}^{0}(\zeta) & f_{\mathrm{q}-1}^{1}(\zeta) & \cdots & \mathrm{f}_{\mathrm{q}-1}^{\mathrm{L}}(\zeta)
\end{array}\right)
$$


The polyphase matrix $\mathcal{P}(\xi)$ is called a unitary matrix if condition (19) holds which is equivalent to

$$
\begin{aligned}
\sum_{\ell=0}^{\mathrm{L}} \overline{\mathrm{f}_{\mathrm{r}}^{\ell}(\zeta)} \mathrm{f}_{\mathrm{r}^{\prime}}^{\ell}(\zeta) & =\delta_{\mathrm{r}, \mathrm{r}^{\prime}} \Leftrightarrow \sum_{\ell=1}^{\mathrm{L}} \overline{\mathrm{f}_{\mathrm{r}^{\prime}}^{\ell}(\zeta)} \mathrm{f}_{\mathrm{r}}^{\ell}(\zeta) \\
& =\delta_{\mathrm{r}, \mathrm{r}^{\prime}}-\overline{\mathrm{f}_{\mathrm{r}}^{0}(\zeta)} \mathrm{f}_{\mathrm{r}^{\prime}}^{0}(\zeta), 0 \leq \mathrm{r}, \mathrm{r}^{\prime} \leq \mathrm{q}-1 .
\end{aligned}
$$

The following theorem, the main result of this article shows that a unitary polyphase matrix leads to a tight wavelet frame on local fields of positive characteristic.

Theorem 1 Suppose that the refinable function $\phi$ and the framelet symbols $\mathrm{m}_{0}, \mathrm{~m}_{1}, \ldots, \mathrm{m}_{\mathrm{L}}$ satisfy equations (8)-(11). Moreover, if the polyphase matrix $\mathcal{P}(\zeta)$ given by (20) satisfy UEP condition (19), then the framelet system $\mathcal{W}(\psi, j, k)$ given by $(5)$ constitutes a tight frame for $\mathrm{L}^{2}(\mathrm{~K})$ i.e.,

$$
\sum_{\ell=1}^{\mathrm{L}} \sum_{j \in \mathbb{Z}} \sum_{k \in \mathbb{N}_{0}}\left|\left\langle f, \psi_{j, k}^{\ell}\right\rangle\right|^{2}=\|f\|_{2}^{2}, \quad \text { for all } f \in \mathrm{L}^{2}(K) .
$$

Proof. By Parseval's formula, we have

$$
\begin{aligned}
\sum_{\ell=1}^{L} \sum_{j \in \mathbb{Z}} \sum_{k \in \mathbb{N}_{0}}\left|\left\langle f, \psi_{j, k}^{\ell}\right\rangle\right|^{2} & =\sum_{\ell=1}^{L} \sum_{j \in \mathbb{Z}} \sum_{k \in \mathbb{N}_{0}}\left|\left\langle f, q^{j / 2} \psi^{\ell}\left(\mathfrak{p}^{-j} x-u(k)\right)\right\rangle\right|^{2} \\
& =\sum_{\ell=1}^{L} \sum_{j \in \mathbb{Z}} \sum_{k \in \mathbb{N}_{0}}\left|\left\langle\hat{f}, q^{j / 2} \hat{\psi}^{\ell}\left(\mathfrak{p}^{-j} \xi\right) x_{\mathfrak{p}^{j}}(\xi)\right\rangle\right|^{2} \\
& =\sum_{\ell=1}^{L} \sum_{j \in \mathbb{Z}} q^{j} \sum_{k \in \mathbb{N}_{0}}\left|\left\langle\hat{f}\left(\mathfrak{p}^{-j} \xi\right) \overline{\hat{\psi}^{\ell}(\xi)}, x(\xi)\right\rangle\right|^{2} \\
& =\sum_{\ell=1}^{L} \sum_{j \in \mathbb{Z}} q^{j} \int_{K}\left|\hat{f}\left(\mathfrak{p}^{-j} \xi\right)\right|^{2}\left|\hat{\psi}^{\ell}(\xi)\right|^{2} d \xi .
\end{aligned}
$$

Using the polyphase decomposition formula (16) of the framelet symbols $\mathrm{m}_{\ell}(\xi), \ell=1, \ldots, \mathrm{L}$, we can write

$$
\sum_{\ell=1}^{\mathrm{L}}\left|\hat{\psi}^{\ell}(\xi)\right|^{2}=\sum_{\ell=1}^{\mathrm{L}}\left|\mathrm{m}_{\ell}(\mathfrak{p} \xi) \hat{\phi}(\mathfrak{p} \xi)\right|^{2}=\sum_{\ell=1}^{\mathrm{L}} \overline{\mathrm{m}_{\ell}(\mathfrak{p} \xi)} \overline{\hat{\phi}(\mathfrak{p} \xi)} \mathrm{m}_{\ell}(\mathfrak{p} \xi) \hat{\phi}(\mathfrak{p} \xi)
$$




$$
\begin{aligned}
& =\overline{\hat{\phi}(\mathfrak{p} \xi)} \sum_{\ell=1}^{\mathrm{L}} \overline{\left\{\frac{1}{\sqrt{\mathrm{q}}} \sum_{\mathrm{r}=0}^{\mathrm{q}-1} \overline{\chi_{\mathfrak{u}(\mathrm{r})}(\mathfrak{p} \xi)} f_{\mathrm{r}}^{\ell}(\zeta)\right\}}\left\{\frac{1}{\sqrt{\mathrm{q}}} \sum_{\mathrm{r}^{\prime}=0}^{\mathrm{q}-1} \overline{\chi_{\mathfrak{u}\left(\mathrm{r}^{\prime}\right)}(\mathfrak{p} \xi)} \mathrm{f}_{\mathrm{r}^{\prime}}^{\ell}(\zeta)\right\} \hat{\phi}(\mathfrak{p} \xi) \\
& =\overline{\hat{\phi}(\mathfrak{p} \xi)} \frac{1}{\mathrm{q}} \sum_{\mathrm{r}=0}^{\mathrm{q}-1} \sum_{\mathrm{r}^{\prime}=0}^{\mathrm{q}-1} \chi_{\mathrm{u}(\mathrm{r})-\mathfrak{u}\left(\mathrm{r}^{\prime}\right)}(\mathfrak{p} \xi)\left\{\sum_{\ell=1}^{\mathrm{L}} \overline{\mathrm{f}_{\mathrm{r}}^{\ell}(\zeta)} \mathrm{f}_{\mathrm{r}^{\prime}}^{\ell}(\zeta)\right\} \hat{\phi}(\mathfrak{p} \xi) .
\end{aligned}
$$

Since the polyphase matrix $\mathcal{P}(\xi)$ is unitary, which is equivalent to condition (21), the above expression reduces to

$$
\begin{aligned}
& \sum_{\ell=1}^{\mathrm{L}}\left|\hat{\psi}^{\ell}(\xi)\right|^{2}=\overline{\hat{\phi}(\mathfrak{p} \xi)} \frac{1}{\mathrm{q}} \sum_{\mathrm{r}=0}^{\mathrm{q}-1} \sum_{\mathrm{r}^{\prime}=0}^{\mathrm{q}-1} \chi_{\mathfrak{u}(\mathrm{r})-\mathfrak{u}\left(\mathrm{r}^{\prime}\right)}(\mathfrak{p} \xi)\left[\delta_{\mathrm{r}, \mathrm{r}^{\prime}}-f_{\mathrm{r}}^{0}(\zeta) f_{\mathrm{r}^{\prime}}^{0}(\zeta)\right] \hat{\phi}(\mathfrak{p} \xi) \\
& =\overline{\hat{\phi}(\mathfrak{p} \xi)} \hat{\phi}(\mathfrak{p} \xi)-\overline{\hat{\phi}(\mathfrak{p} \xi)} \frac{1}{\mathrm{q}} \sum_{\mathrm{r}=0}^{\mathrm{q}-1} \sum_{\mathrm{r}^{\prime}=0}^{\mathrm{q}-1} \chi_{\mathfrak{u}(\mathrm{r})-\mathfrak{u}\left(\mathrm{r}^{\prime}\right)}(\mathfrak{p} \xi) \overline{\mathrm{f}_{\mathrm{r}}^{0}(\zeta)} \mathrm{f}_{\mathrm{r}^{\prime}}^{0}(\zeta) \hat{\phi}(\mathfrak{p} \xi) \\
& =|\hat{\phi}(\mathfrak{p} \xi)|^{2}-\overline{\hat{\phi}(\mathfrak{p} \xi)} \overline{m_{0}(\mathfrak{p} \xi)} \mathrm{m}_{0}(\mathfrak{p} \xi) \hat{\phi}(\mathfrak{p} \xi) \\
& =|\hat{\phi}(\mathfrak{p} \xi)|^{2}-\left|\mathrm{m}_{0}(\mathfrak{p} \xi) \hat{\phi}(\mathfrak{p} \xi)\right|^{2} \\
& =|\hat{\phi}(\mathfrak{p} \xi)|^{2}-|\hat{\phi}(\xi)|^{2} \text {. }
\end{aligned}
$$

Substituting equation (24) in (23), we obtain

$$
\begin{aligned}
\sum_{\ell=1}^{L} \sum_{j \in \mathbb{Z}} \sum_{k \in \mathbb{N}_{0}}\left|\left\langle f, \psi_{j, k}^{\ell}\right\rangle\right|^{2} & =\sum_{j \in \mathbb{Z}} q^{j} \int_{K}\left|\hat{f}\left(\mathfrak{p}^{-j} \xi\right)\right|^{2}\left\{|\hat{\phi}(\mathfrak{p} \xi)|^{2}-|\hat{\phi}(\xi)|^{2}\right\} d \xi \\
& =\int_{K}|\hat{\mathfrak{f}}(\xi)|^{2} \sum_{j \in \mathbb{Z}}\left\{\left|\hat{\phi}\left(\mathfrak{p}^{j+1} \xi\right)\right|^{2}-\left|\hat{\phi}\left(\mathfrak{p}^{j} \xi\right)\right|^{2}\right\} d \xi .
\end{aligned}
$$

Using equation (10), the summand in the above equation can be reformatted as

$$
\begin{aligned}
\sum_{j \in \mathbb{Z}}\left\{\left|\hat{\phi}\left(\mathfrak{p}^{j+1} \xi\right)\right|^{2}-\left|\hat{\phi}\left(\mathfrak{p}^{j} \xi\right)\right|^{2}\right\} d \xi & =\lim _{j \rightarrow \infty}\left|\hat{\phi}\left(\mathfrak{p}^{j+1} \xi\right)\right|^{2}-\lim _{j \rightarrow-\infty}\left|\hat{\phi}\left(\mathfrak{p}^{j} \xi\right)\right|^{2} \\
& =\lim _{j \rightarrow \infty}\left|\hat{\phi}\left(\mathfrak{p}^{j} \xi\right)\right|^{2}-\lim _{j \rightarrow \infty}\left|\hat{\phi}\left(\mathfrak{p}^{-j} \xi\right)\right|^{2} \\
& =|\hat{\phi}(0)|^{2}-\lim _{j \rightarrow \infty}\left|\hat{\phi}\left(\mathfrak{p}^{-j} \xi\right)\right|^{2} \\
& =1 .
\end{aligned}
$$


Using the above estimate in equation (25), we obtain

$$
\sum_{\ell=1}^{\mathrm{L}} \sum_{j \in \mathbb{Z}} \sum_{k \in \mathbb{N}_{0}}\left|\left\langle f, \psi_{j, k}^{\ell}\right\rangle\right|^{2}=\int_{K}|\hat{f}(\xi)|^{2} \mathrm{~d} \xi=\|\hat{\mathrm{f}}\|_{2}^{2}=\|\mathrm{f}\|_{2}^{2} .
$$

This completes the proof of the theorem.

\section{References}

[1] J. J. Benedetto, R. L. Benedetto, A wavelet theory for local fields and related groups, J. Geom. Anal., 14 (2004), 423-456.

[2] I. Daubechies, B. Han, A. Ron, Z. Shen, Framelets: MRA-based constructions of wavelet frames, Appl. Comput. Harmonic Anal., 14 (2003), 1-46.

[3] L. Debnath, F. A. Shah, Wavelet Transforms and Their Applications, Birkhäuser, New York, 2015.

[4] H. K. Jiang, D.F. Li, N. Jin, Multiresolution analysis on local fields, J. Math. Anal. Appl., 294 (2004), 523-532.

[5] J. Krommweh, Tight frame characterization of multiwavelet vector functions in terms of the polyphase matrix, Int. J. Wavelets, Multiresol. Informat. Process., 7 (2009), 9-21.

[6] M. J. Lai, J. Stöckler, Construction of multivariate compactly supported tight wavelet frames, Appl. Comput. Harmonic Anal., 21 (2006), 324-348.

[7] A. P. Petukhov, Explicit construction of framelets, Appl. Comput. Harmonic Anal. 11 (2001), 313-327.

[8] A. Ron, Z. Shen, Affine systems in $\mathrm{L}^{2}\left(\mathbb{R}^{\mathrm{d}}\right)$ : the analysis of the analysis operator, J. Funct. Anal. 148 (1997), 408-447.

[9] F. A. Shah, Frame multiresolution analysis on local fields of positive characteristic, J. Operators. (2015) Article ID 216060, 8 pages.

[10] F. A. Shah, Periodic wavelet frames on local fields of positive characteristic, Numer. Funct. Anal. Optimizat., 37(5) (2016), 603-627.

[11] F. A. Shah, Abdullah, Wave packet frames on local fields of positive characteristic, Appl. Math. Comput., 249 (2014), 133-141. 
[12] F. A. Shah, Abdullah, A characterization of tight wavelet frames on local fields of positive characteristic, J. Contemp. Math. Anal., 49 (2014), 251259.

[13] F. A. Shah, M. Y. Bhat, Tight framelet packets on local fields of positive characteristic, J. Classical Anal., 6 (2015), 85-101.

[14] F. A. Shah, M. Y. Bhat, Semi-orthogonal wavelet frames on local fields, Analysis., 36 (3) (2016), 173-182.

[15] F. A. Shah, L. Debnath, Tight wavelet frames on local fields, Analysis., 33 (2013), 293-307.

[16] M. H. Taibleson, Fourier Analysis on Local Fields, Princeton University Press, Princeton 1975.

Received: August 6, 2016 